\title{
Farewell: retirement as Editor-in-Chief of the Journal of Human Genetics and launching Human Genome Variation
}

Journal of Human Genetics (2014) 59, 2-4; doi:10.1038/jhg.2013.126

First of all, I would like to express my sincere thanks for the great Fhelp and support I have received from the Associate Editors and Editorial Board members, all the reviewers, authors and readers of the Journal of Human Genetics $(J H G)$. The past six years have been very busy but also very valuable to me as Editor-in-Chief of the journal. During this time, JHG has become much more popular around the world and we have published articles submitted from more than 45 different countries. Online visitors to the JHG website have increased by about 2.5 times over last 5 years and I hope our readers enjoyed the publication of our first two special sections on pharmacogenomics and epigenetics last year.

Now it is time to pass my duties to the new Editor-in-Chief Professor Naomichi Matsumoto. I am certain that JHG will continue to grow and become more successful under his direction, and with our new Editorial members.

Currently, I am working hard to launch a new sister journal to $J H G$, Human Genome Variation (HGV) supported by the Japan Society of Human Genetics and published by Nature Publishing

Group. The journal is an Open Access Journal publishing short reports on new mutations/variations of disease-causative genes and their frequencies in various populations in the world, as well as original articles and reviews. I hope this new journal will also be of interest to many of the readers of JHG.

\section{ACKNOWLEDGMENTS}

The Editor-in-Chief on behalf of the Journal of Human Genetics Editorial Board and the Japan Society of Human Genetics, gratefully acknowledges the generous support received from peer reviewers in carefully assessing manuscripts under consideration. The following individuals provided reviews of papers submitted to the Journal of Human Genetics in 2013:

Fukao, Toshiyuki

Fukushima, Kunihiro

Funayama, Manabu

Furukawa, Tetsushi

Ghezzi, Daniele

Goto, Jun

Goto, Yu-ichi

Grønskov, K

Grandone, E

Grasso, Marina

Guan, Min-Xin

\section{Hamada, Hiromichi}

Hasegawa, Tomonobu

Hashimoto, Ryota

Hashimoto, Tamaoki

Hashimoto, Tomoko

Hata, Ken-ichirou

Hattori, Nobutaka

Hayashi, Shin
Katsushi Tokunaga Editor-in-Chief

Journal of Human Genetics
Chaubey, Gyaneshwer

Chen, Zhiyong

Chen, Zhuo

Cherny, Stacey
Fucharoen, Suthat

Fujimoto, Akihiro

Fukami, Maki
Hayashi, Takaaki

Hayashi, Yukiko

Hayde, Karen

Hegele, Robert

Hinney, Anke

Hino, Okio

Hirankarn, Nattiya

Hirawa, Nobuhito

Hirose, Shinichi

Hirota, Tomomitsu

Hoffman, Eric

Horikawa, Yukio

Horike, Shin-ichi

Hosomichi, Kazuyoshi

Hotta, Kikuko

Ikegawa, Shiro

Ikeuchi, Takeshi

Imoto, Issei

Inai, Kei

Inoue, Ituro 
Intarapanich, Apichart

Isaacs, Aaron

Ishida, Takafumi

Ishikawa, Kinya

Ishiura, Hiroyuki

Isomura, Minoru

Ito, Shuichi

Iwamoto, Sadahiko

Iwasaki, Naoko

Jensen-Seaman, Michael I.

Kamatani, Yoichiro

Kaname, Tadashi

Kang, Peter

Kanno, Hitoshi

Kato, Norihiro

Katsanis, Nicholas

Kaur, Gurvinder

Kawame, Hiroshi

Keicho, Naoto

Kent, Lindsey

Ki, Chang-Seok

Kimia, Kahrizi

Kimura, Akinori

Kimura, Ryosuke

Kistner-Griffin, Emily

Kiyotani, Kazuma

Kobayashi, Kazuhiro

Kochi, Yuta

Koene, Saskia

Kohara, Katsuhiko

Kohda, Takashi

Kohno, Takashi

Koizumi, Akio

Kokotas, Haris

Komatsu, Masato

Kondo, Eri

Konta, Tsuneo

Kosaki, Kenjiro

Kowa, Hisatomo

Kozaki, Ken-ichi

Kshatriya, Gautam K.

Kubo, Michiaki

Kubota, Takeo

Kumai, Toshio

Kunugi, Hiroshi

Kurahashi, Hiroki

Kure, Shigeo

Kure, Shigeo

Kurima, Kiyoto

Kurosawa, Kenji

Labrune, Philippe

Landsverk, Megan

Larkin, Emma K

Lee, Michael

Lee, Ming Ta Michael

Lee, Suman

Lertrit, Patcharee

Li, Hui
Li, Mingkun

Lin, Hui-Yi

Maeda, Shiro

Mahasirimongkol,

Surakameth

Mahmutoglu, Saadet

Mano, Shuhei

Markoff, Arseni

Maruo, Yoshihiro

Maruyama, Tetsuo

Mascheretti, Sara

Matsubara, Keiko

Matsuda, Koichi

Matsumoto, Naomichi

Matsuura, Masaaki

McElreavey, Ken

Meisler, Miriam

Minari, Jusaku

Misawa, Kazuharu

Mishima, Hiroyuki

Mitchell, John

Mitsunaga, Shigeki

Miura, Kiyonori

Miyake, Noriko

Miyata, Toshiyuki

Mizuta, Ikuko

Mochizuki, Hideki

Mochizuki, Kazuki

Molano, Jesus

Mori, Seiichi

Morisada, Naoya

Morisaki, Hiroko

Moumni, Imen

Muller-Myhsok, Bertram

Muramatsu, Masaaki

Murayama, Kei

Mushiroda, Taisei

Muto, Kaori

Nagai, Toshiro

Nakagawa, Masanori

Nakamori, Masayuki

Nakamura, Akinori

Nakamura, Kimitoshi

Nakamura, Takahiro

Nakanishi, Akira

Nakanishi, Koichi

Nakayama, Tomohiro

Nanba, Eiji

Nawa, Hiroyuki

Nie, Wei

Niehusmann, Pitt

Niihori, Tetsuya

Nishida, Nao

Nishino, Ichizo

Nishio, Hisahide

Niu, Wenquan

Noguchi, Emiko

Numabe, Hironao

Nunoi, Hiroyuki
Ogata, Tsutomu

Ogawa, Atsushi

Ogimoto, Akiyoshi

Ohashi, Hirofumi

Ohashi, Jun

Ohashi, Toya

Ohata, Takako

Ohira, Miki

Oikawa, Shinichi

Oka, Akira

Okada, Yukinori

Okamoto, Nobuhiko

Okano, Yoshiyuki

Okazaki, Yasushi

Okuyama, Torayuki

Onodera, Osamu

Onouchi, Yoshihiro

Osawa, Haruhiko

Ota, Masao

Otowa, Takeshi

Ott, Jurg

Ozaki, Kouichi

Parson, Walther

Pena, Sergio

Peng, Min-Sheng

Pereira, Luisa

Pithukpakorn, Manop

Prchal, Josef

Ravi Savarirayan, Ravi

Richman, David

Rogers, Jeffrey

Ronchi, Dario

Russo, Antonio

Saha, Krishna

Saito, Shinji

Sakai, Norio

Sakurai, Akihiro

Salas, Antonio

Sasaki, Yasushi

Satake, Wataru

Sato, Noriko

Satta, Yoko

Sawai, Hideaki

Schönberger, Katharina

Schaid, Daniel

Schindler, Detlev

Schurr, Theodore

Seki, Naohiko

Setianingsih, Iswari

Shaw, Philip

Sherman, Stephanie

Shi, Hong

Shibata, Hiroki

Shiga, Kensuke

Shigemizu, Daichi

Shimizu, Atsushi

Shimozawa, Nobuyuki

Shin, Dong-Jik
Shintaku, Haruo

Silveira, Isabel

Soejima, Hidenobu

Solomon, Sheila

Song, Ranran

Song, Zhiyin

Sripichai, Orapan

Stone, Ann

Strom, Charles

$\mathrm{Su}$, Bing

Sugie, Hideo

Suktitipat, Bhoom

Suzuki, Akari

Suzuki, Motoshi

Suzuki, Yoichi

Svanborg, Catharina

Tabara, Yasuharu

Tajima, Atsushi

Tajima, Go

Takahashi, Yohei

Takashima, Hiroshi

Takeda, Atsushi

Takeshima, Yasuhiro

Takiyama, Yoshihisa

Tamiya, Gen

Tamura, Kazuo

Tan, EK

Tei, Kumiko

Terao, Chikashi

Terwisscha van Scheltinga, Afke F.

Tsuchiya, Naoyuki

Tsuji, Shoji

Tsukaguchi, Yasuhiro

Ueda, Hironori

Usami, Shin-ichi

Van Oven, Mannis

Venselaar, Hanka

Vigano, Paola

Wada, Takahito

Wang, Y.M.

Watanabe, Atsushi

Watanabe, Hiroshi

Watanabe, Yoriko

Winge, Marten

Wintle, Richard

$\mathrm{Xu}$, Shuhua

Yamagata, Zentaro

Yamagishi, Hiroyuki

Yamaguchi, Tetsutaro

Yamamoto, Ken

Yamamoto, Toshiyuki

Yamashita, Takayuki

Yang, Jun

Yang, Zhenglin 
Yasuda, Kazuki

Yasui, Kohichiroh

Yasunami, Michio
Yatsu, Keisuke

Yokoyama,

Masatoshi
Yokoyama, Takahiko

Yoshida, Kunihiro

Yoshiura, Koh-ichiro
Zhang, Bao-rong

Zhang, Wen

Ziakas, Panos 